\title{
MULTIQUADRIC PREWAVELETS ON NONEQUALLY SPACED KNOTS IN ONE DIMENSION
}

\author{
M. D. BUHMANN
}

\begin{abstract}
In this paper, we identify univariate prewavelets on spaces spanned by translates of multiquadric functions and other radial basis functions with nonequally spaced centers (or "knots"). Although the multiquadric function and its relations are our prime examples, the theory is sufficiently broad to admit prewavelets from other radial basis function spaces as well.
\end{abstract}

\section{INTRODUCTION}

The theme of this work is the construction of univariate prewavelets that are generated by translates of a radial basis function, where the translates are defined by a sequence of nonequally spaced centers. We first explain why radial basis functions are useful for the construction of prewavelets.

The purpose of wavelet and prewavelet decompositions of univariate squareintegrable functions is to decompose those functions both into their different frequency components, much like Fourier analysis does, as well as with respect to time, which is not possible with standard Fourier analysis. Every such decomposition of a given square-integrable function $f$, say, into a time/frequency series starts with establishing an initial approximation $f_{k}$ to $f$ from a linear space $V_{k}$ that is an element of a nested sequence of spaces

$$
V_{0} \subset V_{1} \subset V_{2} \subset \cdots \subset L^{2}(\mathbb{R})
$$

from which the (pre)wavelets are taken. One requires that

$$
\overline{\bigcup_{j=0}^{\infty} V_{j}}=L^{2}(\mathbb{R}),
$$

so that every $f \in L^{2}(\mathbb{R})$ can be approximated arbitrarily closely in the $L^{2}(\mathbb{R})$ norm by an $f_{k} \in V_{k}$ if $k$ is sufficiently large. Then, orthogonal complements $W_{j} \subset V_{j+1}$ of $V_{j}, j \in \mathbb{Z}_{+}$, are studied. In other words, one seeks spaces $W_{j} \subset V_{j+1}$ such that $W_{j} \perp V_{j}$ and $V_{j} \oplus W_{j}=V_{j+1}$. In fact, the sequence of spaces $(1.1)$ is usually bi-infinite $[2,6,7,9]$, but for the results of this paper it is more appropriate to restrict it to an infinite sequence.

By constructing the $W_{j}$ in the outlined fashion, we get an orthogonal decomposition of $f_{k}$ into an $f_{0} \in V_{0}$ plus a series of $g_{\ell} \in W_{\ell}, \ell=0,1, \ldots, k-1$.

Received by the editor June 29, 1994.

1991 Mathematics Subject Classification. Primary 41A15, 41A30, 42C15, 65D15.

(c)1995 American Mathematical Society 
Therefore, a decomposition of any square-integrable $f$ into mutually orthogonal components, corresponding to the different frequency components in Fourier analysis is found. This can be viewed as a spatial orthogonal decomposition

$$
L^{2}(\mathbb{R})=V_{0}+W_{0}+W_{1}+W_{2}+\cdots .
$$

The prewavelets are functions that generate such $W_{j}$. It is preferable that those prewavelets have local support or that they decay, so that the expansion of $f$ 's components in each $W_{j}$ is a localized decomposition (with respect to time). The prewavelets are called wavelets if they are orthonormal bases of the $W_{j}$ 's, but we are only dealing with prewavelets here. It is always the function $f_{k} \in V_{k}$, which may for instance have been obtained by quasi-interpolation (e.g., Buhmann [5]), that is actually decomposed computationally by the so-called fast wavelet transform. Therefore, it is important to the effectiveness of the decomposition that the spaces $V_{k}$ allow $f_{k}$ to be a sufficiently accurate approximation to $f$, even if $k$ is moderate. This is the point where the efficacy of radial basis functions can be exploited.

We want to explain this point further. Radial basis function methods are generally known to be useful and accurate for the approximation of functions; cf., e.g., the review paper by the author [3], which also includes an extensive list of references to other people's work. The key idea is to approximate from a space spanned by translates of a single function $\varrho$, usually of global support, where the translates take the form $\varrho\left(\left|\cdot-x_{j}\right|\right)$ and the $x_{j}$ are given "centers". The methods were first introduced as interpolatory schemes but, because they were found to provide high-quality approximants, they are now used for many different approximation tools. In this paper, we submit another one in the shape of prewavelets. Radial function methods are easy to implement, and so is the approach proposed here, as we shall see, although special considerations are needed for the matrix computations involved and evaluation of approximants if the amount of data is large (Powell [14]). Among all radial functions currently in use, the multiquadric radial function $\varrho(r)=\sqrt{r^{2}+\gamma^{2}}$ is probably best understood, both theoretically and from a practical point of view, and also it is the one most frequently used, partly by virtue of the variable real parameter $\gamma$. Therefore, we shall focus on it in this article.

Up to now, the most frequent choice for the aforementioned $V_{j}$ are spline spaces with equally spaced knots (Chui and Wang [9] for example), i.e., spaces of piecewise polynomials with knotspacing $2^{-j}$ for the $V_{j}$. These spaces are spanned by B-splines which have compact support. This works well except that the resulting approximations are always of limited smoothness. However, if one takes spaces $V_{j}$ spanned by the multiquadric functions centered at $2^{-j} k$, i.e., $\sqrt{\left(\cdot-2^{-j} k\right)^{2}+\gamma^{2}}, k \in \mathbb{Z}$, then the known theory of radial functions (Buhmann [5] for example) provides essentially the same approximation order as splines and infinite differentiability as well. Sometimes better (spectral) approximation orders are obtained too (Buhmann and Dyn [8]), but this depends strongly on $f$ 's smoothness. The price of these advantages is giving up compact support of the basis functions, but there are generating functions for the $V_{j}$ from radial basis function spaces that decay very fast toward infinity. Such generating functions in the univariate multiquadric setting with scattered center points are found in Powell [13], so that a theory of prewavelets on nonequally spaced 
data may be established for radial basis functions. Therein lies the purpose of this work. We therefore study prewavelets that stem from nested spaces $V_{j}$ spanned by a radial basis function, using in particular the multiquadric function and allowing the centers to be scattered. A very general account of generating prewavelets from shift-invariant spaces (including radial function spaces with gridded centers) is given by de Boor, DeVore and Ron [2], but their technique cannot be applied to scattered data. The main focus of that paper lies in compactly supported prewavelets (mainly from box-spline spaces), which do not exist in spaces spanned by radial functions other than $\phi(r)=r^{2 k+1}, k \in \mathbb{Z}_{+}$, in one dimension.

We will show how to find prewavelets $\psi_{j} \in V_{1}$ that are orthogonal to $V_{0}$ and generate

$$
W_{0}=\left\{\sum_{j=-\infty}^{\infty} c_{j} \psi_{j} \mid c=\left\{c_{j}\right\}_{j=-\infty}^{\infty} \in \ell^{2}(\mathbb{Z})\right\},
$$

with $W_{0} \perp V_{0}$, where $V_{0}$ and $V_{1}$ are spaces generated by nonequally spaced translates of a radial basis function such as the multiquadric function $\varrho(r)=$ $\sqrt{r^{2}+\gamma^{2}}$. Here, $V_{0} \subset V_{1}$ is obtained by demanding that these two spaces be produced by way of two sets of scattered centers, one a subset of the other. The "finer" set generates $V_{1}$, of course. Our principal example is the multiquadric radial function, but the setup in this work is sufficiently general to admit other choices of radial functions.

Once it is demonstrated how $V_{0}, V_{1}$ and the prewavelets that generate $W_{0}$ are found, the same principles can be followed to establish a whole sequence of nested subspaces $V_{j}$ of $L^{2}(\mathbb{R})$ and to decompose the space as in (1.3). Incidentally, wavelets can be obtained from those prewavelets by a standard orthonormalization procedure.

Prior work to this paper are the papers about radial basis function prewavelets due to Buhmann [4], Chui, Ward and Stöckler [10] and Micchelli, Rabut and Utreras [12], which treat the case of equally spaced centers only (some of the preliminary results in the first paper mentioned also apply to scattered centers, and, on the other hand, the results in the third paper apply to the multidimensional setting too).

There now follows a section with preliminaries where the spaces $V_{0}$ and $V_{1}$ are defined. Thereafter, there is a section containing the principal results of this paper, where the prewavelets are explicitly constructed and some of their fundamental properties established. Several examples to which our analysis applies are stated at the end of that section as well.

\section{Preliminaries: The spaces $V_{0}$ AND $V_{1}$}

Let two sequences of real numbers

$$
\mathbf{x}=\left\{\cdots<x_{-1}<x_{0}<x_{1}<x_{2}<\cdots\right\}
$$

and

$$
\underline{\tau}=\left\{\cdots<\tau_{-1}<\tau_{0}<\tau_{1}<\tau_{2}<\cdots\right\}
$$

with $\tau_{2 i-1}=x_{i}, i \in \mathbb{Z}$, be given. We assume that these points satisfy the 
boundedness conditions

$$
\kappa:=\sup _{i \in \mathbb{Z}}\left(\tau_{i+1}-\tau_{i}\right)<\infty, \quad \delta:=\inf _{i \in \mathbb{Z}}\left(\tau_{i+1}-\tau_{i}\right)>0 .
$$

We let $\left\{B_{j}^{\mathbf{c}}\right\}_{j=-\infty}^{\infty}$ and $\left\{B_{j}^{\mathbf{f}}\right\}_{j=-\infty}^{\infty}$ be the sequences of B-splines of degree $n$ on the knot sequences $\mathbf{x}$ and $\underline{\tau}$, with supports $\left[x_{j}, x_{j+n+1}\right]$ and $\left[\tau_{j}, \tau_{j+n+1}\right]$, respectively, normalized to form a partition of unity. Let $\phi \in C(\mathbb{R})$ with $\phi(x) \geqslant 0, x \in \mathbb{R}$,

$$
\phi(x)=O\left(|x|^{-n-1-\varepsilon}\right), \quad x \rightarrow \pm \infty, \quad \text { for some positive } \varepsilon,
$$

a real Fourier transform $\hat{\phi}(t)>0, t \in \mathbb{R}$, and $\int_{-\infty}^{\infty} \phi=1$ be given. For instance, we may take $\phi(t)=\frac{1}{2} \varrho^{\prime \prime}(t)$, where $\varrho(t)$ is the multiquadric function $\sqrt{t^{2}+\gamma^{2}}, n=1$ and $\varepsilon=1$. We consider the functions

$$
\begin{aligned}
C_{j}:=B_{j}^{\mathbf{c}} * \phi, & j \in \mathbb{Z}, \\
F_{j}:=B_{j}^{\mathbf{f}} * \phi, & j \in \mathbb{Z} .
\end{aligned}
$$

In (2.2), * denotes convolution. We immediately record the two important identities

$$
\begin{aligned}
& \sum_{j=-\infty}^{\infty}\left|F_{j}(x)\right|=\sum_{j=-\infty}^{\infty} F_{j}(x) \equiv 1, \\
& \sum_{j=-\infty}^{\infty}\left|C_{j}(x)\right|=\sum_{j=-\infty}^{\infty} C_{j}(x) \equiv 1 .
\end{aligned}
$$

They are a result of the fact that the B-splines form a partition of unity and that the integral of $\phi$ is one. With the example of $\phi$ as suggested above and $n=1, C_{j}$ and $F_{j}$ are second divided differences of the multiquadric function with respect to the sequences $\mathbf{x}$ and $\underline{\tau}$, respectively (Powell [13]). We define

$$
\begin{aligned}
& V_{0}:=\left\{\sum_{j=-\infty}^{\infty} c_{j} C_{j} \mid c=\left\{c_{j}\right\}_{j=-\infty}^{\infty} \in \ell^{2}(\mathbb{Z})\right\}, \\
& V_{1}:=\left\{\sum_{j=-\infty}^{\infty} c_{j} F_{j} \mid c=\left\{c_{j}\right\}_{j=-\infty}^{\infty} \in \ell^{2}(\mathbb{Z})\right\} .
\end{aligned}
$$

We note that if $\phi$ is the $\underline{\delta}$-distribution, then we get the spline spaces especially considered in Buhmann and Micchelli [7]. We shall now prove two elementary facts about these spaces. In the next section, we shall proceed to construct the prewavelets that generate the orthogonal complement $W_{0}$ as in (1.4) of $V_{0}$ in $V_{1}$. We begin with

Proposition 1. The space $V_{0}$ is contained in the space $V_{1}$.

Proof. This has already been discussed in Buhmann [4], but we prove it here for the convenience of the reader. Let $c=\left\{c_{j}\right\}_{j=-\infty}^{\infty} \in \ell^{2}(\mathbb{Z})$. Hence the function $f \in V_{0}$ defined pointwise by

$$
f(x)=\sum_{j=-\infty}^{\infty} c_{j} \int_{-\infty}^{\infty} B_{j}^{\mathbf{c}}(y) \phi(x-y) d y, \quad x \in \mathbb{R},
$$


is defined by an absolutely convergent series and bounded in modulus by $\|c\|_{\infty}$, because $\phi(x-y) \geqslant 0$ and the B-splines form a partition of unity. Now recall that there exists a positive constant $\lambda$ that depends only on $n$ (de Boor [1, p. 37]), so that

$$
\lambda\|c\|_{p} \leqslant\left\|\sum_{j=-\infty}^{\infty} c_{j}\left(\frac{n+1}{x_{j+n+1}-x_{j}}\right)^{1 / p} B_{j}^{\mathrm{c}}\right\|_{p} \leqslant\|c\|_{p}, \quad 1 \leqslant p \leqslant \infty,
$$

where $\|\cdot\|_{p}$ denotes the $\ell^{p}(\mathbb{Z})$ or the $L^{p}(\mathbb{R})$ norm as is appropriate. If only $p=\infty$ is admitted into (2.5), $\lambda$ is bounded below by $2^{-n-2} \sqrt{\pi n} /(2 n+1)$ (this is a recent result due to Scherer; de Boor, private communication). It follows from (2.5) with $p=2$ and from the Cauchy-Schwarz inequality that we may exchange summation and integration in (2.4). It is the same as

$$
f(x)=\int_{-\infty}^{\infty} \sum_{j=-\infty}^{\infty} c_{j} B_{j}^{\mathbf{c}}(y) \phi(x-y) d y,
$$

which can be rewritten by the Curry-Schoenberg theorem (de Boor [1, p. 36]) as

$$
f(x)=\int_{-\infty}^{\infty} \sum_{j=-\infty}^{\infty} \tilde{c}_{j} B_{j}^{\mathbf{f}}(y) \phi(x-y) d y
$$

with $\tilde{c} \in \ell^{2}(\mathbb{Z})$. Hence, reverting back to the form of $f$ with the sum outside the integral, $f \in V_{1}$, as required.

It will be convenient in the sequel to write the spaces $V_{0}$ and $V_{1}$ as $\mathscr{S}_{\mathbf{x}, n, \phi}$ and $\mathscr{S}_{\underline{\tau}, n, \phi}$, respectively, so that $\mathscr{S}_{\mathbf{x}, n, \delta}$, for example, is the space of splines of degree $n$ on the knot sequence $\mathbf{x}$ that are in $L^{2}(\mathbb{R})$ (by $(2.5)$ with $p=2$ ).

Of course it is relevant to our discussion that (1.2) can be satisfied in the present setup. Indeed, we have

Theorem 2. Let $\left\{\underline{\tau}^{k}\right\}_{k=0}^{\infty}$ be a sequence of nested knot sequences in $\mathbb{R}$, i.e., $\underline{\tau}^{0} \subset \underline{\tau}^{1} \subset \cdots$, where the nesting is in the same sense as in $\mathbf{x} \subset \underline{\tau}$, that satisfy

$$
\kappa_{k}:=\sup _{j \in \mathbb{Z}}\left(\tau_{j+1}^{k}-\tau_{j}^{k}\right) \rightarrow 0, \quad k \rightarrow \infty,
$$

and become dense in $\mathbb{R}$ for $k \rightarrow \infty$. Here $\underline{\tau}^{k}=\left\{\tau_{j}^{k}\right\}_{j=-\infty}^{\infty}$. Let $V_{k}=\mathscr{S}_{\underline{\tau}^{k}, n, \phi}$. Then

$$
\overline{\bigcup_{k=0}^{\infty} V_{k}}=L^{2}(\mathbb{R})
$$

Proof. As the Fourier transform is an isometric isomorphism $L^{2}(\mathbb{R}) \rightarrow L^{2}(\mathbb{R})$, we may prove (2.6) in the Fourier domain. Let $\hat{h} \in C_{c}(\mathbb{R})$, where $C_{c}(\mathbb{R})$ denotes the space of compactly supported continuous functions on the reals. This is dense in $L^{2}(\mathbb{R})$. We need to show that $\hat{h}$ can be approximated arbitrarily closely in $L^{2}(\mathbb{R})$ by a function of the form $\sum_{j=-\infty}^{\infty} c_{j} B_{j}^{\hat{\mathrm{f}}[k]} \hat{\phi}$ if $k$ is large enough, where $c=\left\{c_{j}\right\}_{j=-\infty}^{\infty} \in \ell^{2}(\mathbb{Z})$ and where the $\left\{B_{j}^{\mathbf{f},[k]}\right\}_{j=-\infty}^{\infty}$ are the B-splines in $\mathscr{S}_{\underline{\tau}^{k}, n, \underline{\delta}}$. Since $\hat{\phi}(t)>0$, we have $\hat{h} / \hat{\phi} \in L^{2}(\mathbb{R})$ and it can be 
approximated arbitrarily closely by $\sum_{j=-\infty}^{\infty} c_{j} \widehat{B_{j}^{f,[k]}}$ if $k$ is large enough. This shows that the theorem is true.

\section{A PREWAVELET ON NONEQUALLY SPACED CENTERS}

In this section we shall construct prewavelets on nonequally spaced centers with the aid of certain "fundamental functions" of splines of degree $2 n+1$. Precisely, suppose that there exist $d^{\ell}=\left\{d_{k}^{\ell}\right\}_{k=-\infty}^{\infty} \in \ell^{1}(\mathbb{Z}) \backslash\{0\}, \ell \in \mathbb{Z}$, such that

$$
\Psi_{\ell}(x)=\sum_{k=-\infty}^{\infty} d_{k}^{\ell} \int_{-\infty}^{\infty} B_{k}^{\mathbf{f}, 2 n+1}(x-y) \phi(y) d y, \quad x \in \mathbb{R},
$$

where $B_{k}^{\mathbf{f}, 2 n+1} \in \mathscr{S}_{\underline{\tau}, 2 n+1, \underline{\delta}}$ are the B-splines with support $\left[\tau_{k}, \tau_{k+2 n+2}\right]$, gives the identity

$$
\int_{-\infty}^{\infty} \phi\left(y-\tau_{j+n+1}\right) \Psi_{\ell}(y) d y= \begin{cases}1 & \text { if } \ell=j \text { and } n \text { odd }, \\ 1 & \text { if } \ell=j+1 \text { and } n \text { even, } \quad j, \ell \in \mathbb{Z} . \\ 0 & \text { otherwise },\end{cases}
$$

We call these $\Psi_{\ell}$ "fundamental functions" because their construction is related to the use of fundamental functions of interpolation for constructing prewavelets in Chui and Wang [9] and in Buhmann and Micchelli [7]. We define $\psi_{\ell}:=\Psi_{2 \ell}^{(n+1)}$. This is in $V_{1}$ because $(n+1)$ st derivatives of B-splines of degree $2 n+1$ are expressible as finite linear combinations of B-splines of degree $n$ (see Schumaker [15, p. 121], for instance, but recall that the B-splines we use are normalized). In order for the differentiation to result in an absolutely convergent series here, we need that $\tau_{i+1}-\tau_{i}$ is bounded away from zero by $\delta>0$ uniformly in $i$, because the formula for the derivative of a B-spline involves division by differences of $\tau_{i}$ 's. We have already required that this be true.

We assert that the desired orthogonality conditions $\psi_{\ell} \perp V_{0}$ hold, i.e., that

$$
\int_{-\infty}^{\infty} \psi_{\ell}(x) C_{k}(x) d x=0, \quad k, \ell \in \mathbb{Z} .
$$

Together with (1.4), they imply $W_{0} \perp V_{0}$. This orthogonality is settled by a simple argument using integration by parts: Suppose (3.2) holds for a suitable $d^{\ell} \in \ell^{1}(\mathbb{Z})$. Let $s_{\ell}$, for any integer $\ell$, be defined by

$$
s_{\ell}(x):=\frac{d^{n+1}}{d x^{n+1}} \sum_{k=-\infty}^{\infty} d_{k}^{\ell} B_{k}^{\mathbf{f}, 2 n+1}(x), \quad x \in \mathbb{R} .
$$

We get, using the fact that $C_{j}$ and $\Psi_{2 \ell}$ and all their derivatives up to degree $n+1$ are absolutely integrable and vanish at infinity, owing to (2.1) and the B-splines' compact support,

$$
\begin{aligned}
\int_{-\infty}^{\infty} \int_{-\infty}^{\infty} B_{j}^{\mathbf{c}}(x-y) \phi(y) d y \int_{-\infty}^{\infty} s_{2 \ell}(x-y) \phi(y) d y d x \\
=(-1)^{n+1} \int_{-\infty}^{\infty} \sum_{k=-\infty}^{\infty} \mu_{k}^{j} \phi\left(x-x_{k}\right) \Psi_{2 \ell}(x) d x=0, \quad j \in \mathbb{Z}
\end{aligned}
$$


because the $(n+1)$ st derivative of $B_{j}^{c}$ is a finite collection of delta functions $\underline{\delta}\left(\cdot-x_{k}\right), k \in \mathbb{Z}$, with real coefficients $\mu_{k}^{j}$. Before we state our principal theorem, we introduce some notation, namely

$$
\Phi(\theta):=\int_{-\infty}^{\infty} \phi(y) \phi(y-\theta) d y, \quad \theta \in \mathbb{R},
$$

that is, $\Phi=\phi * \phi(-\cdot)$. This is a continuous function because of $(2.1)$.

Theorem 3. The functions (3.1) exist as desired and they are such that $\psi_{\ell} \in V_{1}$ and $V_{1}=V_{0} \oplus W_{0}$, if the three sufficient conditions

$$
\begin{aligned}
& \sup _{j \in \mathbb{Z}} \frac{\tau_{j+2 n+2}-\tau_{j}}{2 n+2} \sup _{t \in(-\infty, \infty)} \sum_{k=-\infty}^{\infty} \phi\left(t-\tau_{k}\right) \\
&<2 \inf _{k \in \mathbb{Z}} \frac{\tau_{k+2 n+2}-\tau_{k}}{2 n+2} \inf _{\theta \in\left(\tau_{k}-\tau_{k+n+1}, \tau_{k+2 n+2}-\tau_{k+n+1}\right)} \Phi(\theta), \\
& \sup _{k \in \mathbb{Z}} \frac{\tau_{2 k+2 n+1}-\tau_{2 k-1}}{n+1} \\
& \quad<2 \min \left\{1, \inf _{k \in \mathbb{Z}} \frac{\left(\tau_{2 k+2 n+1}-\tau_{2 k-1}\right)^{2}}{(n+1)^{2}} \inf _{\theta \in\left[0, \tau_{2 k+2 n+1}-\tau_{2 k-1}\right)} \Phi(\theta)\right\}
\end{aligned}
$$

and

$$
\inf _{k \in \mathbb{Z}} \frac{\tau_{k+2 n+2}-\tau_{k}}{2 n+2} \inf _{\theta \in\left(\tau_{k}-\tau_{k+n+1}, \tau_{k+2 n+2}-\tau_{k+n+1}\right)} \Phi(\theta)>\frac{1}{2}
$$

hold. In other words, the $\psi_{\ell}$ are prewavelets, since they generate $W_{0} \perp V_{0}$ with $V_{1}=V_{0} \oplus W_{0}$. These prewavelets satisfy the summability properties

$$
\sum_{j=-\infty}^{\infty}\left|\psi_{\ell}\left(x-\tau_{j}\right)\right| \leqslant \text { const }<\infty \quad \text { uniformly in } x \in \mathbb{R} \text { and } \ell \in \mathbb{Z}
$$

and

$$
\sum_{\ell=-\infty}^{\infty}\left|\psi_{\ell}(x)\right| \leqslant \text { const }<\infty \quad \text { uniformly in } x \in \mathbb{R} .
$$

It follows from our proof of the theorem that (3.5), (3.6) and (3.7) may be replaced by three weaker but harder-to-verify conditions. (That is the reason why we have opted to mention them now rather than in the statement of the theorem.) In order to state them, it is convenient to adopt yet another notation, viz.

$$
D_{j k}:=\int_{-\infty}^{\infty} \int_{-\infty}^{\infty} \phi\left(y-\tau_{k+n+1}\right) B_{j}^{\mathbf{f}, 2 n+1}(y-z) \phi(z) d z d y, \quad j, k \in \mathbb{Z}
$$

This is well defined because of (2.1). The new conditions are

$$
\begin{gathered}
\sup _{j \in \mathbb{Z}} \sum_{k=-\infty}^{\infty} D_{j k}<2 \inf _{k \in \mathbb{Z}} D_{k k}, \\
\sup _{k \in \mathbb{Z}} \frac{\tau_{2 k+2 n+1}-\tau_{2 k-1}}{n+1}<2 \min \left\{1, \inf _{k \in \mathbb{Z}} \frac{1}{2 \pi} \int_{-\infty}^{\infty}|\hat{\phi}(y)|^{2}\left|\widehat{B_{k}^{\mathrm{c}}}(y)\right|^{2} d y\right\}
\end{gathered}
$$


and

$$
\inf _{k \in \mathbb{Z}} D_{k k}>\frac{1}{2}
$$

respectively. Note that there are sets $\underline{\tau}$ that satisfy these conditions, because, for instance, any small enough pertubation of $\underline{\tau}=\frac{1}{2} \mathbb{Z}$ does so for the multiquadric function $\phi=\frac{1}{2} \varrho^{\prime \prime}, \varrho(r)=\sqrt{r^{2}+\gamma^{2}}, n=1$, if $\gamma$ is small enough. For that choice, the difference between the right- and the left-hand sides of (3.11) is $\approx \frac{1}{3}$ for small $\gamma$, and so is the difference between the right- and the left-hand sides of (3.12). The left-hand side of (3.13) is $\approx \frac{2}{3}$ when $\gamma$ is small.

Proof of Theorem 3. Condition (3.2) means that the $d^{\ell}$ are the $\ell$ th row of the inverse of the bi-infinite matrix $D=\left\{D_{j k}\right\}_{j, k=-\infty}^{\infty}$ when $n$ is odd, otherwise it is the inverse of $D$ shifted by one. This matrix $D$ is a continuous linear operator $D: \ell^{p}(\mathbb{Z}) \rightarrow \ell^{p}(\mathbb{Z})$ for $p \in\{1, \infty\}$ (thus for all $p \in[1, \infty]$ by the Riesz convexity theorem), as we shall see now. Indeed, the matrix $D$ has $\ell^{1}(\mathbb{Z})$ norm one because the B-splines form a partition of unity and because it was a condition on $\phi$ that its integral be one. Further, the matrix $D$ has bounded $\ell^{\infty}(\mathbb{Z})$ norm. This is a consequence of Schumaker's formula [15, p. 128] for the zero moment of a (normalized) B-spline, namely

$$
\|D\|_{\infty} \leqslant \sup _{j \in \mathbb{Z}} \frac{\tau_{j+2 n+2}-\tau_{j}}{2 n+2} \sup _{t \in(-\infty, \infty)} \sum_{k=-\infty}^{\infty} \phi\left(t-\tau_{k}\right) .
$$

The expression on the right-hand side of (3.14) is finite because of $\kappa<\infty$ and (2.1), together with the boundedness condition $\tau_{k+1}-\tau_{k} \geqslant \delta, k \in \mathbb{Z}$. Thus, under the conditions stated in the theorem, $\|D\|_{\infty}<\infty$. If (3.11) instead of (3.5) is demanded, the $\ell^{\infty}(\mathbb{Z})$ boundedness of the operator $D$ becomes, in fact, explicit.

The operator $D$ may be inverted in $\ell^{\infty}(\mathbb{Z})$ by a Neumann series approach, because $\|D-I\|_{\infty}<1$, as we shall now derive from (3.5) (or (3.11)). (Here, $I$ is the identity matrix on $\mathbb{Z} \times \mathbb{Z}$.) To explain this point in detail, observe that there is a $\theta \in\left(\tau_{k}-\tau_{k+n+1}, \tau_{k+2 n+2}-\tau_{k+n+1}\right)$ such that

$$
\begin{aligned}
D_{k k} & =\int_{-\infty}^{\infty} \Phi(z) B_{k}^{\mathbf{f}, 2 n+1}\left(\tau_{k+n+1}+z\right) d z \\
& =\int_{\tau_{k}-\tau_{k+n+1}}^{\tau_{k+2 n+2}-\tau_{k+n+1}} \Phi(z) B_{k}^{\mathbf{f}, 2 n+1}\left(\tau_{k+n+1}+z\right) d y \\
& =\Phi(\theta) \int_{\tau_{k}}^{\tau_{k+2 n+2}} B_{k}^{\mathbf{f}, 2 n+1}(z) d z \\
& =\frac{\tau_{k+2 n+2}-\tau_{k}}{2 n+2} \Phi(\theta) .
\end{aligned}
$$

Nonnegativity of $D$ 's entries and $\|D\|_{1}=1$ imply $D_{k k} \leqslant 1$, and so

$$
\|D-I\|_{\infty} \leqslant\|D\|_{\infty}+1-2 \inf _{k \in \mathbb{Z}} D_{k k} \text {. }
$$

The penultimate display, (3.14), (3.15) and (3.5) give the desired estimate $\|D-I\|_{\infty}<1$. Therefore, $D$ is a homeomorphism on $\ell^{\infty}(\mathbb{Z})$, whence the coefficients $d_{k}^{\ell}$ exist with $\sup _{\ell \in \mathbb{Z}}\left\|d^{\ell}\right\|_{1}<\infty$. That the weaker requirement 
(3.11) may replace (3.5), with $D$ 's invertibility by Neumann series remaining intact, can be seen from (3.15).

It is a direct consequence of $d^{\ell} \in \ell^{1}(\mathbb{Z})$ and our conditions in the statement of the theorem that

$$
\sum_{j=-\infty}^{\infty}\left|\Psi_{\ell}\left(x-\tau_{j}\right)\right| \leqslant \sup _{t \in(-\infty, \infty)} \sum_{j=-\infty}^{\infty} \phi\left(t-\tau_{j}\right) \sup _{\ell \in \mathbb{Z}}\left\|d^{\ell}\right\|_{1} \sup _{k \in \mathbb{Z}} \frac{\tau_{k+2 n+2}-\tau_{k}}{2 n+2}
$$

The right-hand side is finite because the right-hand side of (3.14) is. In order to deduce (3.8) from (3.16), one uses the fact that $(n+1)$ st derivatives of B-splines of degree $2 n+1$ are finite linear combinations of those of degree $n$ (Schumaker [15, p. 121]). Precisely, the left-hand side of (3.8) is bounded above by

$$
\frac{1}{2} \sup _{t \in(-\infty, \infty)} \sum_{j=-\infty}^{\infty} \phi\left(t-\tau_{j}\right) \sup _{\ell \in \mathbb{Z}}\left\|d^{\ell}\right\|_{1} \sup _{k \in \mathbb{Z}} \frac{\tau_{k+n+1}-\tau_{k}}{n+1}\left(\frac{1}{2} \delta\right)^{-n-1}
$$

according to Schumaker's differentiation formula. If $\phi$ is sufficiently differentiable and (2.1) holds also for derivatives of $\phi$ of order at most $n+1$, then the bound (3.17) may be replaced by

$$
\sup _{t \in(-\infty, \infty)} \sum_{j=-\infty}^{\infty}\left|\phi^{(n+1)}\left(t-\tau_{j}\right)\right| \sup _{\ell \in \mathbb{Z}}\left\|d^{\ell}\right\|_{1} \sup _{k \in \mathbb{Z}} \frac{\tau_{k+2 n+2}-\tau_{k}}{2 n+2},
$$

which is a bound that does not contain the increasing factor $\delta^{-n-1}$ as the spacing of the $\tau_{k}$ 's decreases.

We have to show (3.9). By the same arguments as in the penultimate paragraph,

$$
\|D-I\|_{1} \leqslant\|D\|_{1}+1-2 \inf _{k \in \mathbb{Z}} D_{k k} \text {. }
$$

Hence, under our assumption (3.7) or, in its weaker form, (3.13), there holds $\|D-I\|_{1}<1$. So the coefficients $d_{k}^{\ell}$ are also absolutely summable over $\ell \in \mathbb{Z}$. Using the fact that the B-splines $\left\{B_{k}^{f, 2 n+1}\right\}_{k=-\infty}^{\infty}$ form a partition of unity, we may conclude from $d_{k}=\left\{d_{k}^{\ell}\right\}_{\ell=-\infty}^{\infty} \in \ell^{1}(\mathbb{Z}), k \in \mathbb{Z}$, and (2.1) that

$$
\sum_{\ell=-\infty}^{\infty}\left|\Psi_{\ell}(x)\right| \leqslant \sup _{k \in \mathbb{Z}}\left\|d_{k}\right\|_{1} \text {. }
$$

This inequality implies (3.9) because Schumaker's differentiation formula for B-splines supplies once more the explicit bound

$$
\sum_{\ell=-\infty}^{\infty}\left|\psi_{\ell}(x)\right| \leqslant \frac{1}{2} \sup _{k \in \mathbb{Z}}\left\|d_{k}\right\|_{1}\left(\frac{1}{2} \delta\right)^{-n-1} \text {. }
$$

A remark as the one after (3.17) applies here as well: in this case, (3.19) may be replaced by

$$
\sum_{\ell=-\infty}^{\infty}\left|\psi_{\ell}(x)\right| \leqslant \sup _{k \in \mathbb{Z}}\left\|d_{k}\right\|_{1} \int_{-\infty}^{\infty}\left|\phi^{(n+1)}\right| .
$$

The claim $V_{1}=V_{0} \oplus W_{0}$ is established as follows. Recall that every $g \in V_{1}$ is expressible as an infinite linear combination of $F_{j}$ 's with square-summable 
coefficients. We require a decomposition of each $F_{j} \in V_{1}$ first. To this end, we seek coefficients $B_{j k}, j, k \in \mathbb{Z}$, such that the orthogonality conditions

$$
\eta_{j}:=F_{j}-\sum_{k=-\infty}^{\infty} B_{j k} C_{k} \perp C_{\ell}, \quad \ell \in \mathbb{Z},
$$

hold for all integers $j$. In other words, we require that

$$
\sum_{k=-\infty}^{\infty} B_{j k}\left\langle C_{k}, C_{\ell}\right\rangle=\left\langle F_{j}, C_{\ell}\right\rangle, \quad \ell \in \mathbb{Z}
$$

where $\langle\cdot, \cdot\rangle$ is the standard $L^{2}(\mathbb{R})$ inner product. Once such $B_{j k}$ are found, we shall expand $\eta_{j}$ in terms of the $\psi_{k}$ 's to reach the desired conclusion $F_{j} \in$ $V_{0} \oplus W_{0}$.

As the entries of the symmetric matrix $\chi=\left\{\left\langle C_{k}, C_{\ell}\right\rangle\right\}_{k, \ell \in \mathbb{Z}}$ are nonnegative and the B-splines form a partition of unity, the matrix has $\ell^{1}(\mathbb{Z})$ and $\ell^{\infty}(\mathbb{Z})$ norm $\sup _{k \in \mathbb{Z}}\left(\tau_{2 k+2 n+1}-\tau_{2 k-1}\right) /(n+1)$. This is finite because $\kappa$ is. More importantly, it is true that, under condition (3.12) or (3.6), $\|I-\chi\|_{1}=$ $\|I-\chi\|_{\infty}<1$. The specific argument is as follows: First, we note that

$$
\begin{aligned}
\|I-\chi\|_{p} \leqslant & \sup _{k \in \mathbb{Z}} \frac{\tau_{2 k+2 n+1}-\tau_{2 k-1}}{n+1} \\
& +\max \left\{-1,1-2 \inf _{k \in \mathbb{Z}}\left\langle C_{k}, C_{k}\right\rangle\right\}, \quad p=1, \infty,
\end{aligned}
$$

and then

$$
\begin{aligned}
\left\langle C_{k}, C_{k}\right\rangle & =\int_{-\infty}^{\infty} B_{k}^{\mathbf{c}}(z) \int_{-\infty}^{\infty} \Phi(x) B_{k}^{\mathbf{c}}(x+z) d x d z \\
& =\int_{\tau_{2 k-1}}^{\tau_{2 k+2 n+1}} B_{k}^{\mathbf{c}}(z) \int_{-\infty}^{\infty} \Phi(x) B_{k}^{\mathbf{c}}(x+z) d x d z \\
& =\frac{\left(\tau_{2 k+2 n+1}-\tau_{2 k-1}\right)^{2}}{(n+1)^{2}} \Phi(\theta)
\end{aligned}
$$

for a suitable $\theta \in\left[0, \tau_{2 k+2 n+1}-\tau_{2 k-1}\right)$. Thus, according to the conditions in the statement of our theorem, the right-hand side of (3.22) is less than one. Hence $\chi$ is a homeomorphism with inverse $\chi^{-1}$ in $\ell^{1}(\mathbb{Z})$ and in $\ell^{\infty}(\mathbb{Z})$. That the weaker requirement (3.12) is appropriate follows from the display (3.22) and from

$$
\left\langle C_{k}, C_{k}\right\rangle=\frac{1}{2 \pi} \int_{-\infty}^{\infty}|\hat{\phi}(y)|^{2}\left|\widehat{B_{k}^{\mathbf{c}}}(y)\right|^{2} d y
$$

by the Parseval-Plancherel identity. (The latter may be applied by (2.5) [for $p=2$ ] and the integrability of $\phi$.

The desired coefficients are $B_{j k}:=\sum_{\ell \in \mathbb{Z}}\left\langle F_{j}, C_{\ell}\right\rangle \chi_{\ell k}^{-1}$; we denote the matrix $\left\{B_{j k}\right\}_{j, k=-\infty}^{\infty}$ by $B$. The matrix $B$ is well defined and $\|B\|_{1},\|B\|_{\infty}$ are finite because of (2.3) and $\chi$ 's invertibility. Hence, in particular, $\eta_{j} \in V_{1}$ by Proposition 1 and Theorem 275 on p. 198 of Hardy, Littlewood and Pólya [11].

To advance the proof, we require 
Lemma 4. Let $\eta_{j}, j \in \mathbb{Z}$, be defined by (3.20). For each $j$ there exists an $n+1$ times differentiable $H_{j}$ such that $H_{j}^{(n+1)}=\eta_{j}$. Moreover,

$$
\sup _{j \in \mathbb{Z}} \sum_{k=-\infty}^{\infty}\left|\left\langle\phi\left(\cdot-\tau_{2 k}\right), H_{j}\right\rangle\right|<\infty
$$

and

$$
\sup _{k \in \mathbb{Z}} \sum_{j=-\infty}^{\infty}\left|\left\langle\phi\left(\cdot-\tau_{2 k}\right), H_{j}\right\rangle\right|<\infty .
$$

Proof. We note first that there is such an $H_{j}$ for each integer $j$. We can provide it explicitly:

$$
H_{j}(x)=\frac{1}{n !} \int_{-\infty}^{\infty}(x-t)_{+}^{n} \eta_{j}(t) d t, \quad x \in \mathbb{R},
$$

where $(\cdot)_{+}^{n}$ is a truncated power. It follows from $\eta_{j}$ 's definition, $\|B\|_{\infty}<\infty$ and the upper bound (2.1) that $H_{j}$ is well defined. We observe

$$
\left\langle H_{j}, \phi\left(\cdot-\tau_{2 \ell-1}\right)\right\rangle=0, \quad \ell \in \mathbb{Z} .
$$

In order to prove (3.26), we note that, by integration by parts and (3.20),

$$
\begin{aligned}
0 & =\left\langle\eta_{j}, C_{\ell}\right\rangle \\
& =(-1)^{n+1}\left\langle H_{j}, \int_{-\infty}^{\infty} B_{\ell}^{\mathbf{c}^{(n+1)}}(y) \phi(\cdot-y) d y\right\rangle \\
& =\left\langle H_{j}, \sum_{k=-\infty}^{\infty} \mu_{k}^{\ell} \phi\left(\cdot-\tau_{2 k-1}\right)\right\rangle,
\end{aligned}
$$

where the $\left\{\mu_{k}^{\ell}\right\}_{k=-\infty}^{\infty}$ are fixed multiples of the coefficients of the $(n+1)$ st-order divided difference operator based on the knots associated with $B_{\ell}^{\mathrm{c}}$ 's support. The last display implies (3.26) as $\left\langle H_{j}, \phi\left(\cdot-\tau_{k}\right)\right\rangle$ is of polynomial growth but not a polynomial, whereas there are no nontrivial polynomially growing sequences (except polynomials of degree $n$ ) in the kernel of the aforementioned difference operator.

Therefore, by taking an $(n+1)$ st divided difference of $\left\langle\phi(\cdot-y), H_{j}\right\rangle$ with respect to $y$ at the $n+2$ points $\tau_{2 k-1}, \tau_{2 k}, \tau_{2 k+1}, \tau_{2 k+3}, \tau_{2 k+5}, \ldots, \tau_{2 k+2 n-1}$, and by the Peano kernel theorem,

$$
\left|\left\langle\phi\left(\cdot-\tau_{2 k}\right), H_{j}\right\rangle\right| \leqslant C\left|\left\langle\phi\left(\cdot-\tilde{\tau}_{2 k}\right)\left[\tau_{2 k+2 n-1}-\tau_{2 k-1}\right]^{n+1},\left|\eta_{j}\right|\right\rangle\right|
$$

for a suitable positive constant $C$. Here, $\tilde{\tau}_{2 k} \in\left(\tau_{2 k-1}, \tau_{2 k+2 n-1}\right)$. If we sum (3.27) over $j \in \mathbb{Z}$, the result is bounded independently of $k$, since $\sum_{j=-\infty}^{\infty}\left|\eta_{j}\right|$ is uniformly bounded because of (2.1), the compact support of the B-splines and $\|B\|_{1}<\infty$. By the same token, if (3.27) is summed over $k$, the result is again bounded, independently of $j$, since $\eta_{j} \in L^{1}(\mathbb{R})$ and $\kappa<\infty$.

We continue with the proof of Theorem 3 and recall that it is still our aim to expand each $\eta_{j}$ in terms of the $\psi_{k}$ which proves $F_{j} \in V_{0} \oplus W_{0}$. Equipped with Lemma 4, our goal is precisely to show the following lemma: 
Lemma 5. Let $\eta_{j}, j \in \mathbb{Z}$, be defined by (3.20). Then

$$
\eta_{j}=\sum_{k=-\infty}^{\infty} A_{j k} \psi_{k}, \quad j \in \mathbb{Z},
$$

for coefficients $A_{j k}$ which are absolutely summable over $j \in \mathbb{Z}$ as well as over $k \in \mathbb{Z}$.

Proof. We observe immediately that it suffices to prove instead of (3.28) that

$$
\tilde{H}_{j}=\sum_{k=-\infty}^{\infty} A_{j k} \Psi_{2 k}, \quad j \in \mathbb{Z},
$$

for suitable coefficients $A_{j k}$ and for an $\tilde{H}_{j}$ with $\tilde{H}_{j}^{(n+1)}=\eta_{j}$, because we may differentiate on both sides of this identity $(n+1)$ times. We claim that, owing to the form of the $\Psi_{k}$ and the properties of $H_{j}$, the following choice is suitable:

$$
\left.\begin{array}{ll}
\tilde{H}_{j}:=\sum_{k=-\infty}^{\infty}\left\langle\phi\left(\cdot-\tau_{2 k+n+1}\right), H_{j}\right\rangle \Psi_{2 k}, & n \text { odd } \\
\tilde{H}_{j}:=\sum_{k=-\infty}^{\infty}\left\langle\phi\left(\cdot-\tau_{2 k+n}\right), H_{j}\right\rangle \Psi_{2 k}, & n \text { even }
\end{array}\right\}, \quad j \in \mathbb{Z},
$$

the series being absolutely convergent because the $\Psi_{2 k}$ are uniformly bounded by $\sup _{\ell \in \mathbb{Z}}\left\|d^{\ell}\right\|_{1}$ and because of Lemma 4 . Theorem 275 on p. 198 of Hardy, Littlewood and Pólya [11], Lemma 4 and $\left\|d^{\ell}\right\|_{1}<\infty$ imply that the right-hand sides of (3.30) are in $V_{1}^{2 n+1}$, where we let $V_{1}^{2 n+1}=\mathscr{S}_{\underline{\tau}, 2 n+1, \phi}$.

By taking inner products $\langle\cdot, \cdot\rangle$ with $\phi\left(\cdot-\tau_{\ell}\right)$ on both sides of $(3.30)$, we observe that (3.2) implies for odd $n$

$$
\left\langle\phi\left(\cdot-\tau_{2 \ell+n+1}\right), \tilde{H}_{j}\right\rangle=\left\langle\phi\left(\cdot-\tau_{2 \ell+n+1}\right), H_{j}\right\rangle, \quad \ell \in \mathbb{Z},
$$

while (3.26) implies

$$
\left\langle\phi\left(\cdot-\tau_{2 \ell+n}\right), \tilde{H}_{j}\right\rangle=\left\langle\phi\left(\cdot-\tau_{2 \ell+n}\right), H_{j}\right\rangle=0, \quad \ell \in \mathbb{Z} .
$$

There are analogous identities for even $n$. tion

Nonsingularity of $D$ as an operator $\ell^{2}(\mathbb{Z}) \rightarrow \ell^{2}(\mathbb{Z})$ means that the implica-

$$
f \in V_{1}, F \in V_{1}^{2 n+1}, F^{(n+1)}=f,\left\langle\phi\left(\cdot-\tau_{\ell}\right), F\right\rangle=0 \forall \ell \in \mathbb{Z} \quad \Longrightarrow \quad f=0
$$

holds. Hence, $\tilde{H}_{j}^{(n+1)}=H_{j}^{(n+1)}=\eta_{j}$, as required.

We have thus found the coefficients $A_{j k}, j, k \in \mathbb{Z}$. They are not only absolutely summable over $k$, but also over $j$ because of Lemma 4 . Therefore, Lemma 5 is settled.

Now let $g \in V_{1}, g=\sum_{j=-\infty}^{\infty} c_{j} F_{j}$, for $c=\left\{c_{j}\right\}_{j=-\infty}^{\infty} \in \ell^{2}(\mathbb{Z})$. Then, according to Lemma 5 ,

$$
g=\sum_{k=-\infty}^{\infty} \sum_{j=-\infty}^{\infty} c_{j} A_{j k} \psi_{k}+\sum_{k=-\infty}^{\infty} \sum_{j=-\infty}^{\infty} c_{j} B_{j k} C_{k}
$$

with

$$
\sum_{k=-\infty}^{\infty}\left(\sum_{j=-\infty}^{\infty} c_{j} A_{j k}\right)^{2}<\infty, \quad \sum_{k=-\infty}^{\infty}\left(\sum_{j=-\infty}^{\infty} c_{j} B_{j k}\right)^{2}<\infty
$$


The second inequality in (3.31) follows from the fact that $\sum_{k=-\infty}^{\infty}\left|B_{j k}\right| \leqslant$ const $<\infty$ and $\sum_{j=-\infty}^{\infty}\left|B_{j k}\right| \leqslant$ const $<\infty$, uniformly in $j$ and $k$, respectively, and from Theorem 275 on p. 198 of Hardy, Littlewood and Pólya [11]. The first inequality in (3.31) is true by Lemma 5.

We remark that the assertions of Theorem 3 remain true if $\phi$ is no longer positive, so long as it is explicitly demanded that $\|D-I\|_{\infty}<1,\|D-I\|_{1}<1$ and $\|\chi-I\|_{\infty}<1$, in order that the Neumann series argument can be applied.

Theorem 6. Given the setup of Theorems 2 and 3, there are prewavelets $\left\{\psi_{\ell k}\right\}_{\ell=-\infty}^{\infty}, k \in \mathbb{Z}_{+}$, in $V_{k+1}$ and spaces $W_{k}$ spanned by sums of those prewavelets with square-summable coefficients such that $V_{k+1}=V_{k} \oplus W_{k}, k \in \mathbb{Z}_{+}$, and

$$
L^{2}(\mathbb{R})=V_{0} \oplus \bigoplus_{k=0}^{\infty} W_{k},
$$

if the assumptions of Theorem 3 hold for all $\underline{\tau}^{k}$.

Proof. Let $f \in L^{2}(\mathbb{R})$ and let a positive $\varepsilon$ be given. In view of Theorem 2, there are $k \in \mathbb{Z}_{+}$and $f_{k} \in V_{k}$ such that

Further,

$$
\left\|f-f_{k}\right\|_{2}^{2} \leqslant \varepsilon \text {. }
$$

$$
f_{\ell+1}=f_{\ell}+g_{\ell}, \quad f_{\ell} \in V_{\ell}, \quad g_{\ell} \in W_{\ell}, \quad \forall 0 \leqslant \ell<k .
$$

Therefore, the arbitrariness of $\varepsilon>0$ and the orthogonality between different $W_{\ell}$ 's imply (3.32).

We point out that, according to the requirements of Theorem 3 , it may not always be possible to construct prewavelets that span $W_{k}$ for arbitrarily large $k$ depending on $\underline{\tau}^{k}$, the prescribed radial function and its parameters. If this happens in practice and when using multiquadrics for instance, $\gamma$ must be chosen suitably to allow that the sufficient conditions for the existence of prewavelets with small stepsizes in Theorem 3 are met, where the stepsize should be according to the resolution we wish to obtain.

Further examples to our theory can be obtained by applying it, for example, to $\left(r^{2}+\gamma^{2}\right)^{\frac{1}{2} \beta}$ (where $\beta \in \mathbb{Z} \backslash 2 \mathbb{Z}_{+}$). Quickly decaying radial functions with positive Fourier transforms $\phi(r)=\exp \left(-\gamma^{2} r^{2}\right), \gamma>0, \phi(r)=\exp (-\gamma|r|)$, etc., can be used too, in this case directly as $\phi$ without any differentiation. The essential feature that admits derivation of suitable decaying functions from the radial functions by differentiation is that $\hat{\varrho}$ be positive and integrable outside a neighborhood of the origin, with perhaps an integer-order singularity at zero. It is the purpose of the differentiation to resolve this singularity. More specifically, $\varrho(r)={\sqrt{r^{2}+\gamma^{2}}}^{3}$ with $\phi=\varrho^{(4)}$ or $\phi(r)=\frac{1}{r^{2}+\gamma^{2}}$ are both suitable choices for $n=3$ and $n=0$, respectively, except they have to be normalized to integrate to one.

It is highly relevant to the computation of the prewavelets that, if $\phi=\varrho^{(n+1)}$ as in our initial multiquadric example for $n=1$, the entries of the matrix $D$ are a constant multiple of

$$
\int_{-\infty}^{\infty} \varrho\left(y-\tau_{j}\right) \phi_{*}^{k}(y) d y
$$




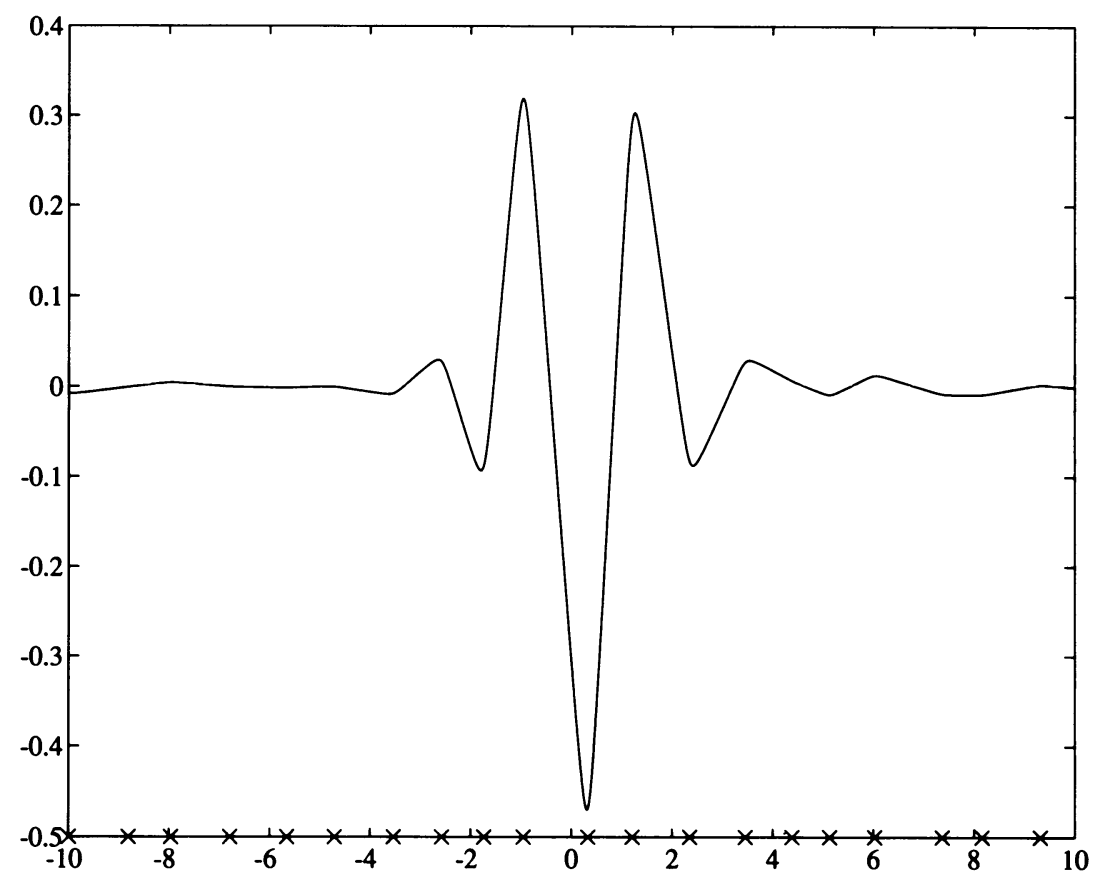

FIGURE 1. Multiquadric prewavelet for $n=1, \gamma=0.1$ and scattered centers

where $\phi_{*}^{k}$ is a $(2 n+2)$ nd-order divided difference of the function $\varrho$ based on the centers that are associated with $B_{k}^{\mathbf{f}, 2 n+1}$ 's support. The formula (3.33) is derived from (3.10) by integration by parts. By the same token, the prewavelets themselves can be expressed as constant multiples of

$$
\sum_{k=-\infty}^{\infty} d_{k}^{2 \ell} \phi_{*}^{k}(y), \quad y \in \mathbb{R}
$$

instead of (3.1). This observation has been used to create Figure 1 of the multiquadric prewavelet for $n=1$. It uses integer data randomly perturbed (by at most 0.4 ) and $\gamma=0.1$. The positions of the centers are indicated by crosses. So we see there a multiquadric prewavelet for scattered centers, probably for the first time in the literature. It remains to add, however, that the theory presented here is far from complete, which reflects the fact that the approach to scattered data prewavelets is substantially different from that of prewavelets on gridded data. For instance, no Riesz basis properties are shown of the prewavelets presented here nor are there concrete estimates for their asymptotic behavior at infinity.

In order not to end on a downbeat note, we point out that it was shown in Buhmann [3] how to derive explicit decay estimates for prewavelets and decomposition coefficients $A_{j k}, B_{j k}$, if $\phi$ is a derivative of a radial basis function from the class discussed in that paper and the centers are equally spaced. In this context it is also important to note that, when the centers are equally spaced, Riesz stability for the $\ell^{2}(\mathbb{Z})$ norm can be shown, so that the prewavelets here form a Riesz basis for $W_{0}$. We do not elaborate on this point, however, because 
the subject of this paper are prewavelets on nonequally spaced centers. (This claim is actually easily established using (2.5) and the fact that $D$ is a homeomorphism on $\ell^{2}(\mathbb{Z})$ - the latter follows from $D$ being a homeomorphism on $\ell^{p}(\mathbb{Z}), p \in\{1, \infty\}$, and from the Riesz convexity theorem.) The subject of Riesz stability will be one of the main topics in the forthcoming paper (Buhmann [6]).

\section{BIBLIOGRAPHY}

1. C. de Boor, Odd-degree spline interpolation at a biinfinite knot sequence, Approximation Theory Bonn 1976 (R. Schaback and K. Scherer, eds.), Lecture Notes in Math., vol. 556, Springer-Verlag, Berlin, 1976, pp. 30-53.

2. C. de Boor, R.A. DeVore, and A. Ron, On the construction of multivariate (pre)wavelets, Constr. Approx. 9 (1993), 123-166.

3. M. D. Buhmann, New developments in the theory of radial basis function interpolation, Multivariate Approximation: From CAGD to Wavelets (K. Jetter and F.I. Utreras, eds.), World Scientific, Singapore, 1993, pp. 35-75.

4. __ Discrete least squares approximation and pre-wavelets from radial function spaces, Math. Proc. Cambridge Philos. Soc. 114 (1993), 533-558.

5. $\_$, On quasi-interpolation with radial basis functions, J. Approx. Theory 72 (1993), 103130.

6. _ Pre-wavelets on scattered knots and from radial function spaces: A review, Proc. 6th Conference on the Mathematics of Surfaces (R.R. Martin, ed.), IMA Conference Proceedings Series, Oxford Univ. Press (to appear).

7. M. D. Buhmann and C.A. Micchelli, Spline prewavelets with non-uniform knots, Numer. Math. 61 (1992), 455-474.

8. M. D. Buhmann and N. Dyn, Error estimates for multiquadric interpolation, Curves and Surfaces (P.J. Laurent, A. LeMéhauté, and L.L. Schumaker, eds.), Academic Press, Boston, 1991, pp. 51-58.

9. C. K. Chui and J.Z. Wang, A cardinal spline approach to wavelets, Proc. Amer. Math. Soc. 113 (1992), 785-793.

10. C. K. Chui, J.D. Ward, and J. Stöckler, Analytic wavelets generated by radial functions, manuscript, 1994.

11. G. H. Hardy, J. E. Littlewood, and G. Pólya, Inequalities, Cambridge Univ. Press, Cambridge, 1934.

12. C. A. Micchelli, C. Rabut, and F. Utreras, Using the refinement equation for the construction of pre-wavelets III: Elliptic splines, Numer. Alg. 1 (1991), 331-352.

13. M. J. D. Powell, Univariate multiquadric interpolation: reproduction of linear polynomials, Multivariate Approximation and Interpolation (W. Haussmann and K. Jetter, eds.), Birkhäuser Verlag, Basel, 1990, pp. 227-240.

14. __ Tabulation of thin plate splines on a very fine two-dimensional grid, Numerical Methods of Approximation Theory (D. Braess and L.L Schumaker, eds.), Birkhäuser, Basel, 1992, pp. 221-244.

15. L. L. Schumaker, Spline functions: Basic theory, Wiley, New York, 1981.

Mathematik Departement, ETH Zentrum, 8092 Zürich, Switzerland

E-mail address: mdb@math.ethz.ch 